\title{
Bi-exponential T2* analysis of healthy and diseased Achilles tendons: an in vivo preliminary magnetic resonance study and correlation with clinical score
}

\author{
Vladimir Juras • Sebastian Apprich • Pavol Szomolanyi • \\ Oliver Bieri • Xeni Deligianni • Siegfried Trattnig
}

Received: 6 February 2013 /Revised: 9 April 2013 / Accepted: 15 April 2013 /Published online: 13 June 2013

(C) The Author(s) 2013. This article is published with open access at Springerlink.com

\begin{abstract}
Objective To compare mono- and bi-exponential $\mathrm{T}_{2}{ }^{*}$ analysis in healthy and degenerated Achilles tendons using a recently introduced magnetic resonance variable-echo-time sequence (vTE) for $\mathrm{T}_{2} *$ mapping.

Methods Ten volunteers and ten patients were included in the study. A variable-echo-time sequence was used with 20 echo times. Images were post-processed with both techniques, mono- and bi-exponential $\left[\mathrm{T}_{2}{ }^{*}\right.$, short $\mathrm{T}_{2}{ }^{*}$ component $\left(\mathrm{T}_{2}{ }_{\mathrm{s}}\right)$ and long $\mathrm{T}_{2} *$ component $\left(\mathrm{T}_{2}{ }_{1}\right)$ ]. The number of mono- and biexponentially decaying pixels in each region of interest was expressed as a ratio $(\mathrm{B} / \mathrm{M})$. Patients were clinically assessed
\end{abstract}

V. Juras $(\bowtie) \cdot$ S. Apprich $\cdot$ P. Szomolanyi $\cdot$ S. Trattnig

Center of Excellence for High field MR, Department of Radiology,

Medical University of Vienna, Waehringer Guertel 18-20, A-1090

Vienna, Austria

e-mail: vladimir.juras@meduniwien.ac.at

S. Apprich

e-mail: sebastian.apprich@meduniwien.ac.at

P. Szomolanyi

e-mail: pavol.szomolanyi@meduniwien.ac.at

S. Trattnig

e-mail: siegfried.trattnig@meduniwien.ac.at

V. Juras $\cdot$ P. Szomolanyi

Department of Imaging Methods, Institute of Measurement

Science, Dubravska cesta 9, 84104 Bratislava, Slovakia

O. Bieri $\cdot X$. Deligianni

Division of Radiological Physics, Department of Radiology,

University of Basel Hospital, Basel, Switzerland

O. Bieri

e-mail: oliver.bieri@unibas.ch

X. Deligianni

e-mail: xeni.deligianni@unibas.ch with the Achilles Tendon Rupture Score (ATRS), and these values were correlated with the $T_{2} *$ values.

Results The means for both $\mathrm{T}_{2}{ }^{*} \mathrm{~m}$ and $\mathrm{T}_{2}{ }^{*}$ s were statistically significantly different between patients and volunteers; however, for $\mathrm{T}_{2}{ }_{\mathrm{s}}$, the $P$ value was lower. In patients, the Pearson correlation coefficient between ATRS and $\mathrm{T}_{2}{ }^{*} \mathrm{~s}$ was -0.816 $(P=0.007)$.

Conclusion The proposed variable-echo-time sequence can be successfully used as an alternative method to UTE sequences with some added benefits, such as a short imaging time along with relatively high resolution and minimised blurring artefacts, and minimised susceptibility artefacts and chemical shift artefacts. Bi-exponential $\mathrm{T}_{2}{ }^{*}$ calculation is superior to mono-exponential in terms of statistical significance for the diagnosis of Achilles tendinopathy.

Key Points

- Magnetic resonance imaging offers new insight into healthy and diseased Achilles tendons

- Bi-exponential T2* calculation in Achilles tendons is more beneficial than mono-exponential

- A short T2* component correlates strongly with clinical score

- Variable echo time sequences successfully used instead of ultrashort echo time sequences

Keywords Achilles tendon · Bi-exponential · T2* A ATRS · Mono-exponential $\cdot$ Magnetic resonance imaging

\section{Introduction}

It is generally known that biophysical properties of the tissue define the contrast between tissues in magnetic resonance imaging (MRI). In addition to field strength and temperature, relaxation times also depend on the macromolecular content 
of the tissue. In highly organised tissues, such as tendons, ligaments, menisci, trabecular bone, or cartilage, the transversal relaxation time is extremely short compared with that of muscles or fat, for instance. Therefore, special MR sequences are required to acquire signal directly from these tissues. In the last 10 years, the most frequently used sequences for quantitative imaging of fast-relaxing tissues comprised the 3D ultrashort echo time (UTE) [1], the 2D-UTE [2], the stack of spirals (AWSOS) [3], and a variable echo time sequence [4, 5]. Macromolecular content causes strong anisotropy in these tissues through the dipole-dipole interaction. In the past, different degrees of anisotropy were observed in various tissues using spectroscopic methods; e.g. in tendons, four peaks were observed in the $T_{2}$ spectrum and in the cartilage two were observed [6,7]. The recent developments in MR hardware and software now allow a quantification of multiple $\mathrm{T}_{2}\left(\right.$ or $\mathrm{T}_{2}{ }^{*}$ ) components in the clinical environment. These can be subsequently used as markers for different pathophysiological conditions. In recently published studies, $\mathrm{T}_{2}{ }^{*}$ has been used as a marker for sub-clinical changes in menisci after an anterior cruciate ligament tear [8]. In other studies, osteoarthritis progression in cartilage was detected using $\mathrm{T}_{2} / \mathrm{T}_{2} *$ [9-11]. As for tendons, several studies have demonstrated the suitability of quantitative $\mathrm{T}_{2}{ }^{*}$ mapping in the Achilles tendon $[12,13]$. Generally, two types of water exist in connective tissues, free water and bound water, where the water molecules can be bound to collagen fibres or proteoglycan molecules. A common problem of bi-component $\mathrm{T}_{2}$ * analysis is that the shortest component is difficult to quantify with clinically common echo times (i.e. TE $>10 \mathrm{~ms}$ ). Another problem is a relatively high sensitivity to the signal-to-noise ratio (SNR) during multiple-component analysis. In our study, a 3D gradient echo (GRE) sequence with a variable echo time (vTE) was used. This sequence, which was introduced by Ying and Schmalbrock [5], and further developed by Song and Wehrli [4] and recently by Deligianni et al. [14], utilises varying phase-encoding gradients to manipulate the effective echo time, resulting in sub-millisecond TE that enables the visualisation of fast-relaxing tissues. The advantage of UTE sequences is the sampling of the MR signal with very short echo times, which allows $T_{2} / T_{2} *$ to be calculated quite precisely. However, low resolution and blurring artefacts complicate $\mathrm{T}_{2} / \mathrm{T}_{2}{ }^{*}$ estimation. The vTE offers images with high resolution without blurring. To the best of our knowledge, this sequence has not been previously used for $\mathrm{T}_{2}{ }^{*}$ calculation in the human Achilles tendon in vivo.

Therefore, the aim of this study was to confirm the suitability of vTE for $\mathrm{T}_{2}{ }^{*}$ estimation in the human Achilles tendon in vivo. Furthermore, we hypothesise that the echo time range provided by vTE would be suitable for biexponential $\mathrm{T}_{2} *$ calculation. We also compared the monoand bi-exponentially calculated $\mathrm{T}_{2} *$ to demonstrate that the short and long $\mathrm{T}_{2} *$ components might help in diagnosing a degenerated Achilles tendon better than simple monoexponentially calculated $\mathrm{T}_{2} *$. To validate this, quantitative MRI data were correlated with clinical scores for Achilles tendon rupture.

\section{Materials and methods}

Patient cohort

Institutional Review Board approval and written, informed

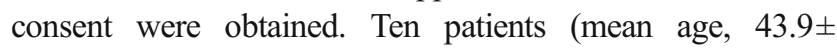
13.4 years) with a painful Achilles tendon and ten agematched, healthy volunteers (mean age, $43.7 \pm 11.2$ years) were included in the study. The exclusion criteria for all subjects were contraindications to MR imaging.

\section{MRI examination}

All subjects were examined with a 3-T whole-body system (TIM Trio, Siemens, Erlangen, Germany) using an eightchannel knee coil (In Vivo, Gainesville, FL, USA). For quantitative bi-exponential $\mathrm{T}_{2}{ }^{*}$ assessment, a multi-echo, variable echo time (me-vTE) sequence was obtained, with sequentially shifted echo times. In total, there were five sets with four echo times each. There were 20 echo times: $\mathrm{TE}=0.8,2.1,3.1,4.1$, 5.1, 6.1, 7.1, 8.1, 9.1, 10.1, 11.1, 12.1, 13.1, 14.1, 15.1, 16.1, 17.1, 18.1, 19.1, and $20.0 \mathrm{~ms}$. Other parameters were set as follows: field of view $118 \times 180 \mathrm{~mm}$, matrix $168 \times 256$, section/slice thickness $0.7 \mathrm{~mm}, 320 \mathrm{~Hz} /$ pixel bandwidth, and 144 sections, resulting in a total acquisition time of $12.16 \mathrm{~min}$. For morphological evaluation of the Achilles tendon, the following set of four sequences was used: fat-saturated sagittal proton-density weighted turbo-spin echo (sag PD TSE), axial $\mathrm{T}_{2}$-weighted TSE (ax T2 TSE), fat-saturated axial PDweighted TSE (ax PD TSE), and a fat-saturated sagittal $\mathrm{T}_{1}$ weighted sequence (sag T1). The parameters for these sequences are listed in Table 1.

\section{Clinical tendon scoring}

All subjects were rated according to the Achilles tendon Total Rupture Score (ATRS; 0-100 points, worst to best) [15]. The ATRS scoring sheet is summarised in Table 2. An orthopaedic surgeon with 5 years of experience (N.N.) rated the tendons.

Image analysis

Magnetic resonance imaging parameters were calculated using a manually drawn ROI analysis in the three regions of the Achilles tendon (insertion part, INS; middle part, MID; muscle-tendon junction, MTJ; Fig. 1). The length of each of the parts was defined as one-third of the total Achilles tendon 
Table 1 Parameters for the sequences used for morphological evaluation of the Achilles tendon

TE echo time, TR repetition time, PAT parallel acquisition techniques, GRAPPA generalised autocalibrating partially parallel acquisitions

\begin{tabular}{lllll}
\hline & sag PD TSE & ax T2 TSE & ax PD TSE & sag T1 \\
\hline Orientation & Sagittal & Axial & Axial & Sagittal \\
TE [ms] & 26 & 98 & 27 & 11 \\
TR [ms] & 3970 & 6720 & 3520 & 600 \\
FOV read [mm] & 220 & 170 & 170 & 220 \\
PAT mode & GRAPPA & GRAPPA & GRAPPA & GRAPPA \\
Acceleration factor & 2 & 2 & 2 & 2 \\
Flip angle $\left[{ }^{\circ}\right]$ & 120 & 120 & 120 & 70 \\
Averages & 1 & 1 & 3 & 3 \\
Slices & 17 & 30 & 30 & 17 \\
Slice thickness [mm] & 2 & 4 & 4 & 2 \\
Bandwidth [Hz/px] & 149 & 177 & 149 & 160 \\
Matrix & $448 \times 381$ & $448 \times 448$ & $896 \times 896$ & $512 \times 512$ \\
Total acq. time [min:s] & $5: 46$ & $3: 22$ & $3: 49$ & $2: 48$ \\
\hline
\end{tabular}

length, measured from the most proximal to the most distal. Values were also stored for the sum of all three regions, hereafter referred to as the 'bulk' values. Images from the vTE sequence were analysed using a custom-written script in IDL 6.3 (Interactive Data Language, Research Systems, Inc., Boulder, CO, USA). A mono- as well as a bi-exponential fitting procedure was performed on all MR data sets on a pixel-by-pixel basis. For mono-exponential fitting, a threeparametric function was used to fit the signal intensity

$\mathrm{S}_{\mathrm{m}}=\mathrm{A}_{0} * \exp \left(-\mathrm{TE} / \mathrm{A}_{1}\right)+\mathrm{A}_{2}$ where $\mathrm{A}_{0}$ is the signal intensity at a $\mathrm{TE}<1 \mathrm{~ms}, \mathrm{~A}_{1}$ corresponds to the actual $\mathrm{T}_{2}{ }_{\mathrm{m}}$ (mono-exponentially calculated $\mathrm{T}_{2}{ }^{*}$ ), and $\mathrm{A}_{2}$ is the baseline (mostly the noise). The same data set was also processed bi-exponentially, using the function

$\mathrm{S}_{\mathrm{b}}=\mathrm{B}_{0} * \exp \left(-\mathrm{TE} / \mathrm{B}_{1}\right)+\mathrm{B}_{2} * \exp \left(-\mathrm{TE} / \mathrm{B}_{3}\right)+\mathrm{B}_{4}$

where $B_{1}$ corresponds to the short component of $T_{2} *\left(T_{2}{ }_{s}\right)$, $\mathrm{B}_{3}$ corresponds to the long component of $\mathrm{T}_{2} *\left(\mathrm{~T}_{2}{ }^{*}\right)$, and $\mathrm{B}_{0}$ and $\mathrm{B}_{2}$ are the component ratios expressed further as a percentage value of $\mathrm{B}_{0}+\mathrm{B}_{2}: \mathrm{Fs}=100 * \mathrm{~B}_{0} /\left(\mathrm{B}_{0}+\mathrm{B}_{2}\right)$ and $\mathrm{F}_{1}=$ $100 * B_{2} /\left(B_{0}+B_{2}\right)$. $B_{4}$ is the offset given primarily by noise.
Table 2 Achilles Tendon Total Rupture Score (ATRS) sheet (the numbers mean level of limitation) [15]

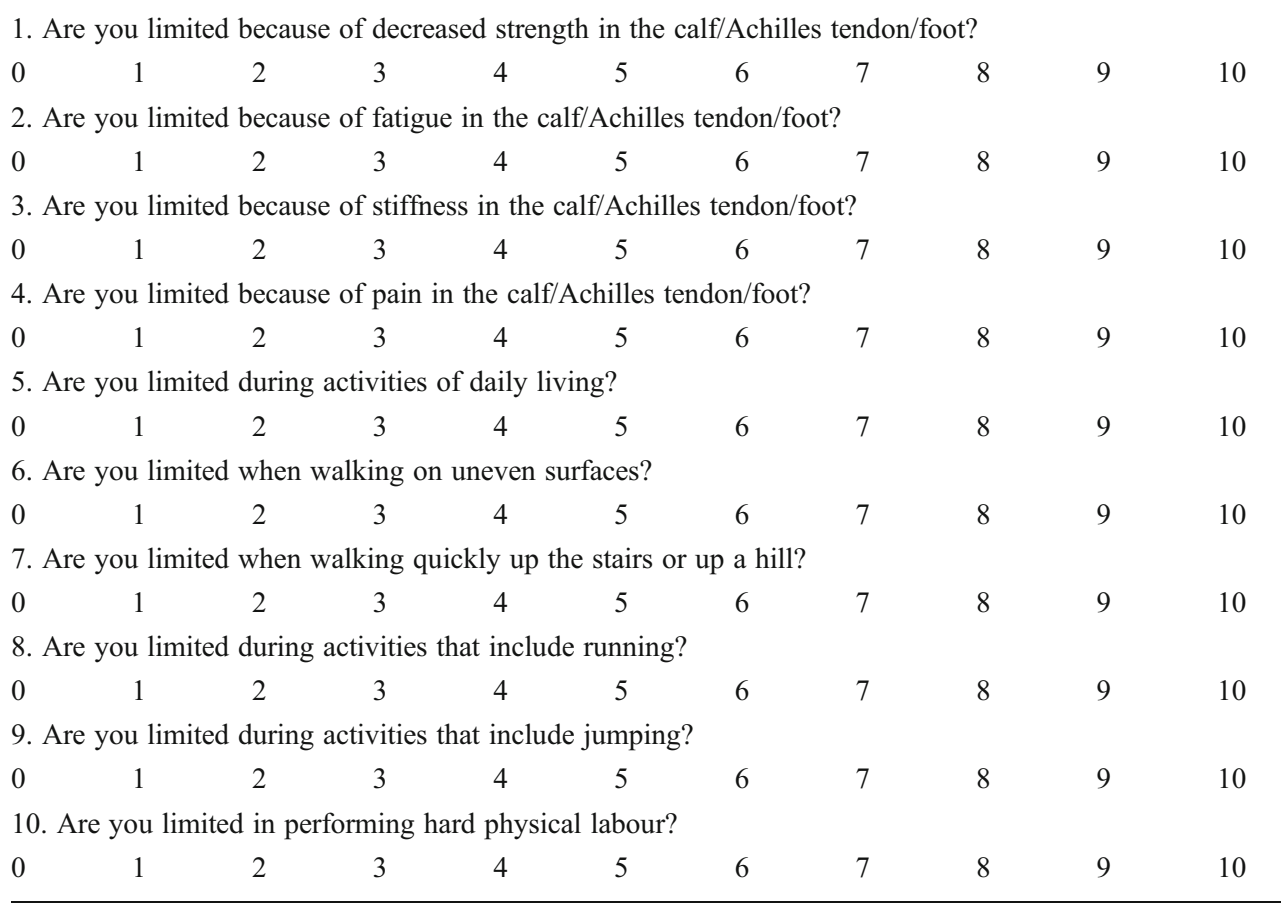




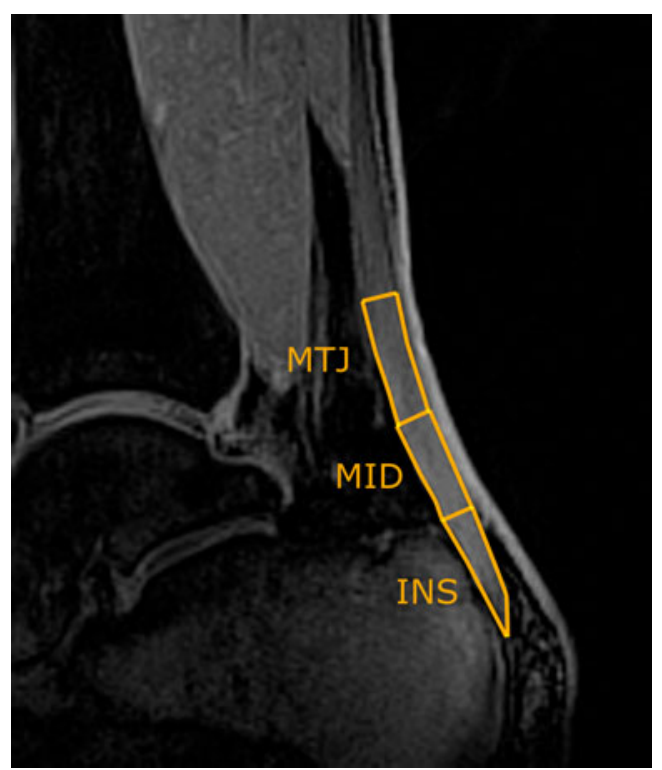

Fig. 1 Region-of-interest placement on vTE image (calculated as a subtraction of images acquired at $\mathrm{TE}=0.8 \mathrm{~ms}$ and $\mathrm{TE}=20 \mathrm{~ms}$ )

During the calculation of $\mathrm{T}_{2}{ }^{*}$, only those pixels that satisfied the following condition were considered bi-exponential:

$4 \times \mathrm{T}_{2} *_{\mathrm{s}}<\mathrm{T}_{2} *_{1}$

Seven maps for each slice were stored for further use, i.e. the mono-exponential $\mathrm{T}_{2}{ }^{*}$ maps, the short component of the bi-exponential $\mathrm{T}_{2}{ }^{*}$ maps, the long component of the biexponential $\mathrm{T}_{2} *$ maps, and both short and long $\mathrm{T}_{2}{ }^{*}$ component ratios and the binary maps of mono- or biexponentially calculated pixels. The map of the coefficient of determination $\left(\mathrm{R}^{2}\right.$, fit precision) and the binary map based on the bi-exponential condition (Eq.3) $(0=$ monoexponential decay, 1 = bi-exponential decay) were saved as well.

Each ROI was represented by a weighted mean value, calculated using an $\mathrm{R}^{2}$ correction algorithm [16]. $\mathrm{T}_{2}{ }_{\mathrm{s}}$ and $\mathrm{T}_{2}{ }^{*}{ }_{1}$ were calculated using a mask created from a binary map, and only pixels that satisfied the condition $4 \times \mathrm{T}_{2}{ }_{\mathrm{s}}<$ $\mathrm{T}_{2}{ }_{1}{ }_{1}$ were included. From each segment, the ratio of monoand bi-exponential pixel amounts was calculated (further referred to as $\mathrm{B} / \mathrm{M})$.

Intra- and interobserver variation

To calculate the interobserver variability, all ten patients were evaluated by three independent raters (V.J., S.A., P.S.). The variation between raters was expressed as a coefficient of variation $(\mathrm{CV}, \%)$. Intraobserver variability was calculated from three independent evaluations of ten patients by one rater (V.J.) and expressed as an intraclass correlation (ICC).
Statistical analysis

All statistical analyses were performed in SPSS for Windows, version 16.0 (SPSS Institute, Chicago, IL, USA). Descriptive statistics were performed in order to calculate the mean and standard deviation (SD) of age, $\mathrm{T}_{2}{ }_{\mathrm{m}}, \mathrm{T}_{2}{ }_{\mathrm{s}}, \mathrm{T}_{2}{ }_{1}$, component ratios, $\mathrm{B} / \mathrm{M}$ values, and ATRS separately for normal and degenerative Achilles tendons. An independent sample $t$-test with equal variances was performed to obtain the difference in individual parameters between the two groups. A $P$ value $<0.05$ was considered statistically significant. The correlation of MR parameters and ATRS was calculated as a Pearson correlation coefficient. A binary classification test was performed on the data to obtain the discrimination power using ROC analyses. The area under the curve was calculated by using the trapezoidal rule and expressed as percentages.

\section{Results}

The coefficient of variation (CV) for interobserver variation was found to be $8.2 \%$, on average. For different tendon segments, the $\mathrm{CV}$ was $5.1 \%$ for the insertion part of the tendon, $7.9 \%$ for the mid part, and $11.8 \%$ for the muscle-tendon junction. Intraobserver variation was expressed using ICC and found to be 0.881 , on average. For different segments, ICC was 0.912 for the insertion part of the tendon, 0.845 for the mid part, and 0.887 for the muscle-tendon junction.

The mean ATRS was $52.8 \pm 23.7$ for patients; all volunteers had an ATRS equal to 100. The mean pixel count for bulk ROIs was $307 \pm 134$; separately, for the individual tendon parts, the INS was $106 \pm 48$, the MID $114 \pm 54$, and the MTJ $87 \pm 64$. The model with the bi-exponential fitting function calculated the values with slightly higher precision than that with a mono-exponential fitting function $\left(\mathrm{R}^{2}[\mathrm{mono}]=0.965 \pm\right.$ $\left.0.144 ; \mathrm{R}^{2}[\mathrm{bi}]=0.996 \pm 0.131\right)$. The data with the corresponding mono- and bi-exponential fits are depicted in Fig. 2. As for results from bulk analysis, in volunteers, the mean $\mathrm{T}_{2}{ }_{\mathrm{m}} \mathrm{m}$ was $3.35 \pm 0.45 \mathrm{~ms}$, the mean $\mathrm{T}_{2}{ }^{*} \mathrm{~s}$ was $0.68 \pm 0.05 \mathrm{~ms}$, the mean $\mathrm{T}_{2}{ }^{*}$ was $16.99 \pm 7.11 \mathrm{~ms}$, and the $\mathrm{B} / \mathrm{M}$ ratio was $7.29 \pm 2.30$. In patients, the mean $\mathrm{T}_{2}{ }_{\mathrm{m}}$ was $6.56 \pm 1.70 \mathrm{~ms}$, the mean $\mathrm{T}_{2}{ }^{*} \mathrm{~s}$ was $0.87 \pm 0.08 \mathrm{~ms}$, the mean $\mathrm{T}_{2}{ }_{1}$ was $19.83 \pm 7.13 \mathrm{~ms}$, and the $\mathrm{B} / \mathrm{M}$ ratio was $3.39 \pm 1.27$. The means for both $\mathrm{T}_{2}{ }_{\mathrm{m}}$ and $\mathrm{T}_{2}{ }_{\mathrm{s}}$ were statistically significantly different between the patients and volunteers; however, the $P$ value for $\mathrm{T}_{2}{ }^{*} \mathrm{~s}$ was substantially lower $(P<0.001)$ than that of $\mathrm{T}_{2}{ }_{\mathrm{m}}(P=0.001)$. Moreover, the amount of bi-exponentially decaying pixels decreased with pathology $(P=0.012)$. All MR parameters, ratios, as well as $P$ values are summarised in Table 3 . The area under the curve was 0.669 for $\mathrm{T}_{2}{ }_{\mathrm{m}}, 0.638$ for $\mathrm{T}_{2}{ }_{\mathrm{s}}, 0.580$ for $\mathrm{T}_{2}{ }_{1}$, and 0.643 for $\mathrm{B} / \mathrm{M}$. Examples of the maps from a volunteer and a patient are depicted in Figs. 3 and 4. 
In patients, the Pearson correlation coefficient between ATRS and $\mathrm{T}_{2}{ }^{*} \mathrm{~s}$ was $r=-0.846(P=0.002)$. High correlation, albeit lower compared with $\mathrm{T}_{2}{ }^{*}$, was also observed between ATRS and $\mathrm{T}_{2}{ }_{\mathrm{m}}(r=-0.786, P=0.007)$. ATRS did not correlate with $\mathrm{T}_{2}{ }_{1}(r=-0.216, P=0.55)$ or $\mathrm{B} / \mathrm{M}(r=-0.02, P=0.956)$ (Fig. 5).

\section{Discussion}

The results of this study suggest that the variable echo time sequence based on a gradient echo is suitable for bicomponent analysis of $\mathrm{T}_{2} *$ in the human Achilles tendon in vivo. Mono-exponential calculation of $\mathrm{T}_{2} *$ provides a weighted mean value from different components of transversal relaxation. This may lead to an underestimation of $\mathrm{T}_{2}{ }^{*}$, especially in diseased tendons. From a biochemical point of view, the short component of $\mathrm{T}_{2}{ }^{*}$ is related to bound water and the long component to free water [17]. This is due to the existence of distinct water compartments with different transverse relaxation times in highly organised biological tissues [18]. $\mathrm{T}_{2}$ and $\mathrm{T}_{2}{ }^{*}$ in tendons have been studied previously using various methods, such as MR spectroscopy, a standard Carr Purcell Meiboom Gill (CPMG) sequence, 2D sequences, and 3D UTE sequences. Peto et al. studied different water compartments in the Achilles tendon ex vivo using MR spectroscopy [19]. They demonstrated four water components by measuring four $\mathrm{T}_{2}{ }^{*}$ components, $1.1,6.0,18.9$, and $79.8 \mathrm{~ms}$, with the component ratios of $56,26,15$, and $3 \% . \mathrm{T}_{2} *$ is substantially dependent on temperature and orientation to the main magnetic field [19]. Henkelman et al. also demonstrated fourcomponent $\mathrm{T}_{2}$ decay in the Achilles tendon using a CMPG technique [6]. Despite this fact, there are many studies using mono-exponential $\mathrm{T}_{2} / \mathrm{T}_{2} *$ calculations that have resulted in a linear combination of short and long $\mathrm{T}_{2} / \mathrm{T}_{2} *$ components. Gold et al. used a projection-reconstruction approach to calculating $\mathrm{T}_{2}$ in ex vivo Achilles tendons; the mean $\mathrm{T}_{2}$ calculated in that study was $1.2 \pm 0.2 \mathrm{~ms}$ [20]. In another study, $\mathrm{T}_{2} *$ was calculated mono-exponentially using a 2D-UTE sequence in ex vivo samples [21], and it ranged from 1.76 to $2.64 \mathrm{~ms}$ for the global average area of the Achilles tendon. A bi-component analysis of transversal relaxation constants in the Achilles tendon is more sensitive to the precision of pixels used for fitting and is also more computationally demanding; however, it allows quantification of the bound and free water. The Graeme Bydder group performed the pioneer work in this field, mostly using 2D-UTE imaging and UTE spectroscopy imaging for fast-relaxing tissues [13, 17, 21, 22]. In a recent study by Diaz et al., investigators observed the different $\mathrm{T}_{2}{ }^{*}$ values in normal and abnormal Achilles tendons, with a short $\mathrm{T}_{2} *$ of $0.61 \pm 0.06 \mathrm{~ms}$ and a long $\mathrm{T}_{2} *$ of $8.23 \pm 1.29$, and a short $\mathrm{T}_{2} *$ of $0.65 \pm 0.04 \mathrm{~ms}$ and a long $\mathrm{T}_{2} *$ of $4.72 \pm 0.77 \mathrm{~ms}$ in normal and abnormal tendons, respectively [23]. Du et al. described methods for bi-component analysis in a comprehensive study of bovine samples. They found two components of $\mathrm{T}_{2} *$ in the Achilles tendon (1.28 \pm 0.08 and $\left.17.65 \pm 4.91 \mathrm{~ms}\right)$ and pointed out the importance of bi-component $\mathrm{T}_{2} *$ measurement in other tissues, such as ligaments, bone, meniscus, and even cartilage [17]. Robson et al. obtained regionally dependent $\mathrm{T}_{2}{ }^{*}$ values of 0.53 and $4.8 \mathrm{~ms}$ in the posterior part and 0.60 and $4.2 \mathrm{~ms}$ in the anterior part, and they observed a reduction in short $\mathrm{T} 2$ components in tendinopathy [13]. Juras et al. used 3D-UTE for bi-component calculation of $\mathrm{T}_{2} *$ and
Fig. 2 Example of mono- and bi-exponential fit of data. The solid line represents the biexponential fit of the data, where $\mathrm{T}_{2}{ }^{*}$ s was $0.88 \mathrm{~ms}$ and $\mathrm{T}_{2}{ }^{*}$ was $25.58 \mathrm{~ms}$ (with component ratios of 56 and $44 \%$, respectively). The dashed line represents the monoexponential fit of the data, where $\mathrm{T}_{2}{ }^{*}$ m was $6.83 \mathrm{~ms}$. $\mathrm{R}^{2}$ was 0.9989 for the biexponential fit and 0.9120 for mono-exponential

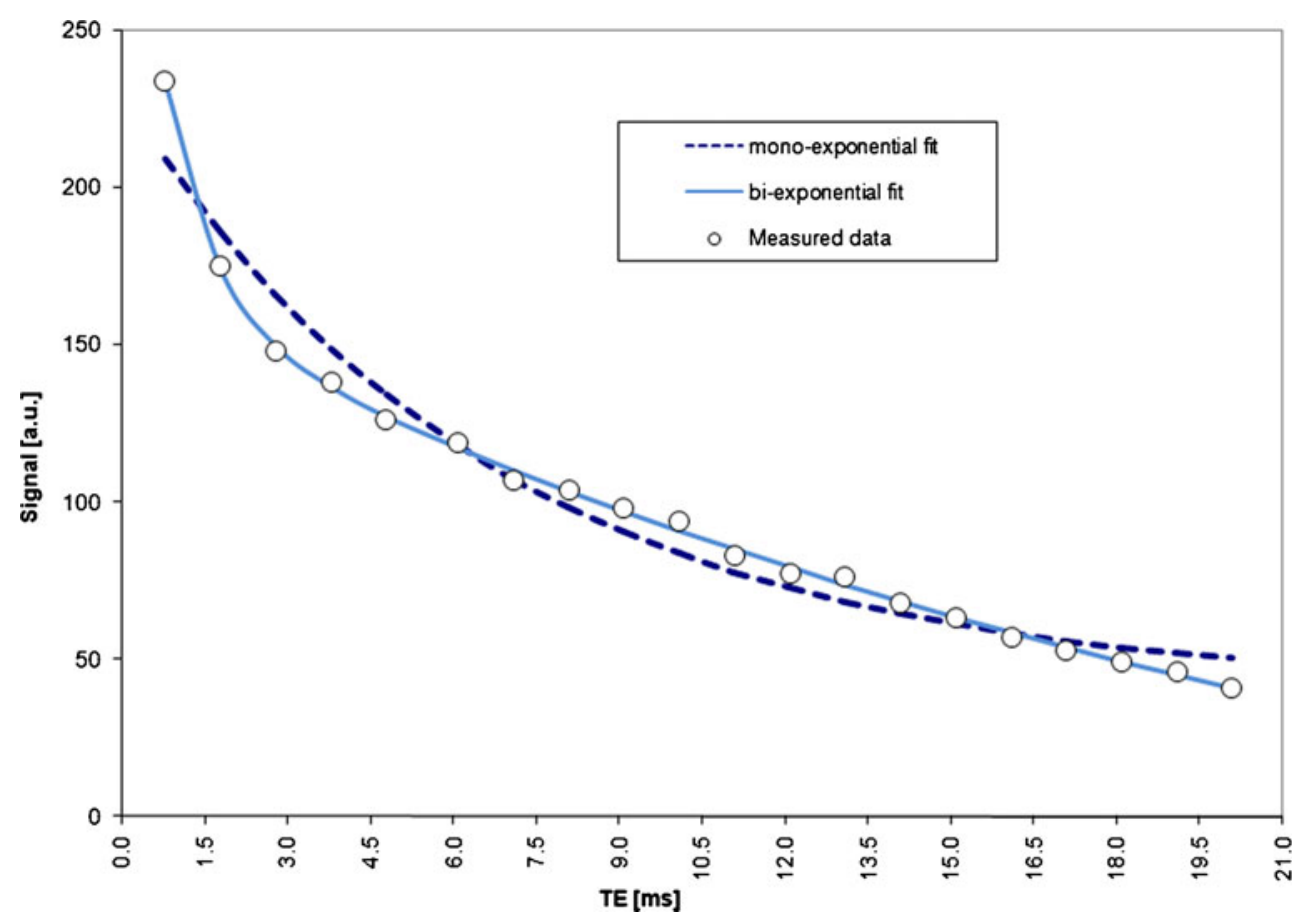


Table 3 Summary of MR parameters in volunteers and patients, and statistical significance of the mean difference in different tendon structures

Asterisk indicates statistical significance $(P<0.05)$

\begin{tabular}{|c|c|c|c|c|c|c|}
\hline & \multirow[t]{2}{*}{ Region } & \multicolumn{2}{|c|}{ Volunteers } & \multicolumn{2}{|c|}{ Patients } & \multirow[t]{2}{*}{$P$ values } \\
\hline & & Mean & Standard deviation & Mean & Standard deviation & \\
\hline \multirow[t]{4}{*}{$\mathrm{T}_{2}{ }_{\mathrm{m}}$} & Bulk & 3.35 & 0.45 & 6.56 & 1.70 & $0.001 *$ \\
\hline & INS & 3.45 & 0.49 & 5.32 & 0.77 & $0.049^{*}$ \\
\hline & MID & 4.42 & 1.16 & 8.91 & 1.88 & $0.033^{*}$ \\
\hline & MTJ & 2.17 & 0.39 & 5.47 & 1.51 & $0.021^{*}$ \\
\hline \multirow[t]{4}{*}{$\mathrm{T}_{2}{ }_{\mathrm{s}}$} & Bulk & 0.68 & 0.05 & 0.87 & 0.08 & $<0.001^{*}$ \\
\hline & INS & 0.68 & 0.05 & 0.95 & 0.16 & $0.006^{*}$ \\
\hline & MID & 0.78 & 0.29 & 0.97 & 0.13 & 0.097 \\
\hline & MTJ & 0.58 & 0.17 & 0.67 & 0.13 & 0.183 \\
\hline \multirow[t]{4}{*}{$\mathrm{T}_{2} *_{1}$} & Bulk & 16.99 & 7.11 & 19.83 & 7.13 & 0.538 \\
\hline & INS & 13.49 & 3.80 & 16.87 & 6.47 & 0.264 \\
\hline & MID & 17.37 & 3.10 & 23.41 & 6.21 & 0.279 \\
\hline & MTJ & 20.13 & 5.21 & 19.21 & 7.67 & 0.082 \\
\hline \multirow[t]{4}{*}{ Short component } & Bulk & 58 & 10 & 60 & 9 & 2.150 \\
\hline & INS & 56 & 11 & 61 & 8 & 0.259 \\
\hline & MID & 56 & 11 & 61 & 9 & 0.144 \\
\hline & MTJ & 57 & 12 & 62 & 6 & 0.078 \\
\hline \multirow[t]{4}{*}{ Long component } & Bulk & 42 & 11 & 40 & 9 & 2.150 \\
\hline & INS & 39 & 8 & 39 & 7 & 0.259 \\
\hline & MID & 44 & 11 & 39 & 9 & 0.144 \\
\hline & MTJ & 43 & 12 & 38 & 6 & 0.078 \\
\hline \multirow[t]{4}{*}{$\mathrm{B} / \mathrm{M}$} & Bulk & 7.29 & 2.30 & 3.39 & 1.27 & $0.012 *$ \\
\hline & INS & 4.26 & 1.91 & 2.62 & 1.73 & 0.291 \\
\hline & MID & 7.96 & 2.89 & 2.64 & 1.29 & 0.157 \\
\hline & MTJ & 9.67 & 2.61 & 4.92 & 2.24 & $0.04 *$ \\
\hline
\end{tabular}

found significant differences between normal and degenerated Achilles tendons $(0.34 \pm 0.09$ and $10.28 \pm 2.28 \mathrm{~ms}$ versus 0.71 \pm 0.17 , and $12.85 \pm 1.87 \mathrm{~ms}$ ) [12]. Those authors found a significant increase in the short $\mathrm{T}_{2} *$ component in degenerative tendon tissue. However, these numbers were measured at $7 \mathrm{~T}$; therefore, they may differ from those measured in this study at $3 \mathrm{~T}$. Noticeably, the variation in the long $\mathrm{T}_{2} *$ component among the studies is much greater than that of the short component, which can provide an explanation for the lower clinical specificity and sensitivity of $\mathrm{T}_{2}{ }_{1}$.

Interestingly, when comparing the $\mathrm{T}_{2}{ }^{*} \mathrm{~m}$ and $\mathrm{T}_{2}{ }_{\mathrm{s}}$ from the whole tendon between volunteers and patients, both

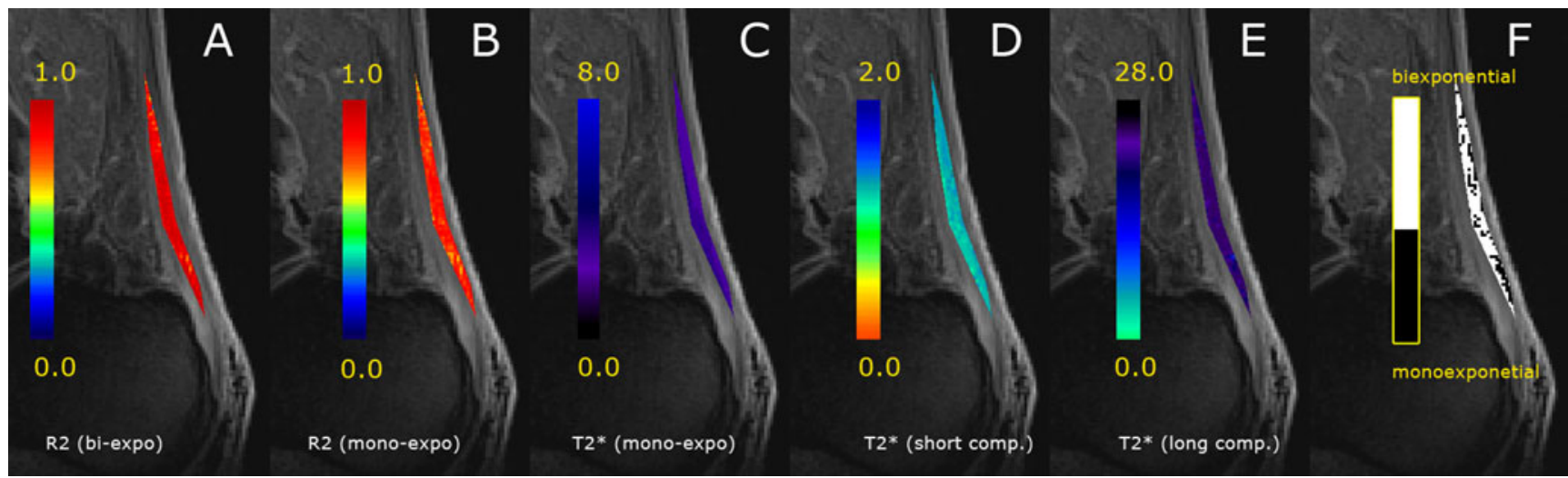

Fig. 3 Example of bi-component $\mathrm{T}_{2} *$ analysis of a 23 -year-old healthy volunteer. a $\mathrm{R}^{2}$ map for bi-exponential $\mathrm{T}_{2} *$ calculation; $\mathbf{b} \mathrm{R}^{2}$ map for mono-exponential $\mathrm{T}_{2} *$ calculation; $\mathbf{c}$ mono-exponentially calculated $\mathrm{T}_{2} *$; d short component of $\mathrm{T}_{2} *$ map; e long component of $\mathrm{T}_{2} *$ map; $\mathbf{f}$ the binary map of mono- and- bi-exponential pixels used for B/M ratio calculation. Individual maps are overlaid on vTE images, calculated as a subtraction of the shortest TE $(0.8 \mathrm{~ms})$ and the longest TE $(20 \mathrm{~ms})$. Note the higher $\mathrm{R}^{2}$ values for bi-exponential calculation 


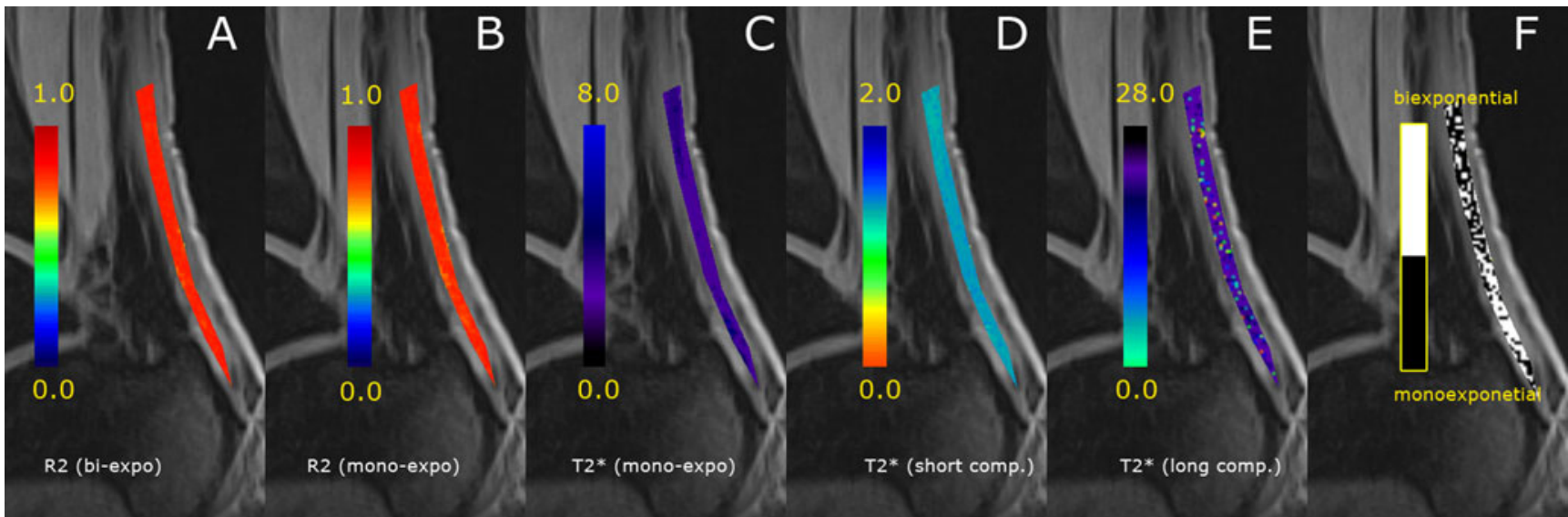

Fig. 4 Example of bi-component $\mathrm{T}_{2} *$ analysis of a 64-year-old patient with an ATRS of 45 . a $\mathrm{R}^{2}$ map for bi-exponential $\mathrm{T}_{2}{ }^{*}$ calculation; $\mathbf{b} \mathrm{R}^{2}$ map for mono-exponential $\mathrm{T}_{2}{ }^{*}$ calculation; $\mathbf{c}$ mono-exponentially calculated $\mathrm{T}_{2} *$; $\mathbf{d}$ short component of $\mathrm{T}_{2}{ }^{*}$ map; e long component of $\mathrm{T}_{2}{ }^{*}$ map; $\mathbf{f}$ the binary map of mono- and- bi-exponential pixels used for $\mathrm{B} /$

mean differences were statistically significant. However, when looking at the separately segmented regions (INS, MID, and MTJ), the difference in $\mathrm{T}_{2}{ }^{*} \mathrm{~m}$ was statistically significant in all parts, while the difference in $\mathrm{T}_{2}{ }_{\mathrm{s}}$ was significant only in the insertion part. This can be explained by the fact that collagen fibre organisation is higher from the MTJ toward the INS; therefore, more pixels were excluded from bi-exponential analysis from the MTJ and MID parts of the tendon, which could have affected the statistics.

To the best of our knowledge, quantitative MRI values have not been correlated with the clinical scores, to date. The ATRS score has high clinical utility, being both patientand clinician-friendly, and it has been validated in many studies [15, 24-26]. Although it was originally developed for measuring outcome after the treatment of total Achilles tendon rupture, it is also extendable to the general evaluation
$\mathrm{M}$ ratio calculation. Individual maps are overlaid on vTE images, calculated as a subtraction of the shortest TE $(0.8 \mathrm{~ms})$ and the longest TE $(20 \mathrm{~ms})$. Note the higher diversity of the long $\mathrm{T}_{2} *$ component and bi-exponential pixels, as well as the altered ratio of mono- and biexponentially calculated pixels compared to volunteer's data

of the Achilles tendon status. The high correlation of $\mathrm{T}_{2}{ }^{*} \mathrm{~m}$ and $\mathrm{T}_{2}{ }^{*} \mathrm{~s}$ with clinical scores in our study validates the clinical value of quantitative MRI for Achilles tendons.

The intra- and interobserver variability showed a very high reproducibility for the evaluation. The highest variability was found in the MTJ part of the tendon, which is probably attributable to the more difficult segmentation in the region where the tendon connects to the soleus and gastrocnemius muscles.

Our study has several limitations. Possible patient movement may have introduced an error in the sensitive biexponential $\mathrm{T}_{2}{ }^{*}$. Movement of the subjects was minimised by careful ankle fixation, and the images were also coregistered in the post-processing phase.

Some bias may have also been introduced by choosing the constant in the condition for bi-exponentially decaying pixels, which was based purely on empirical findings. This choice seemed to best fit the mathematical model of bi-exponential
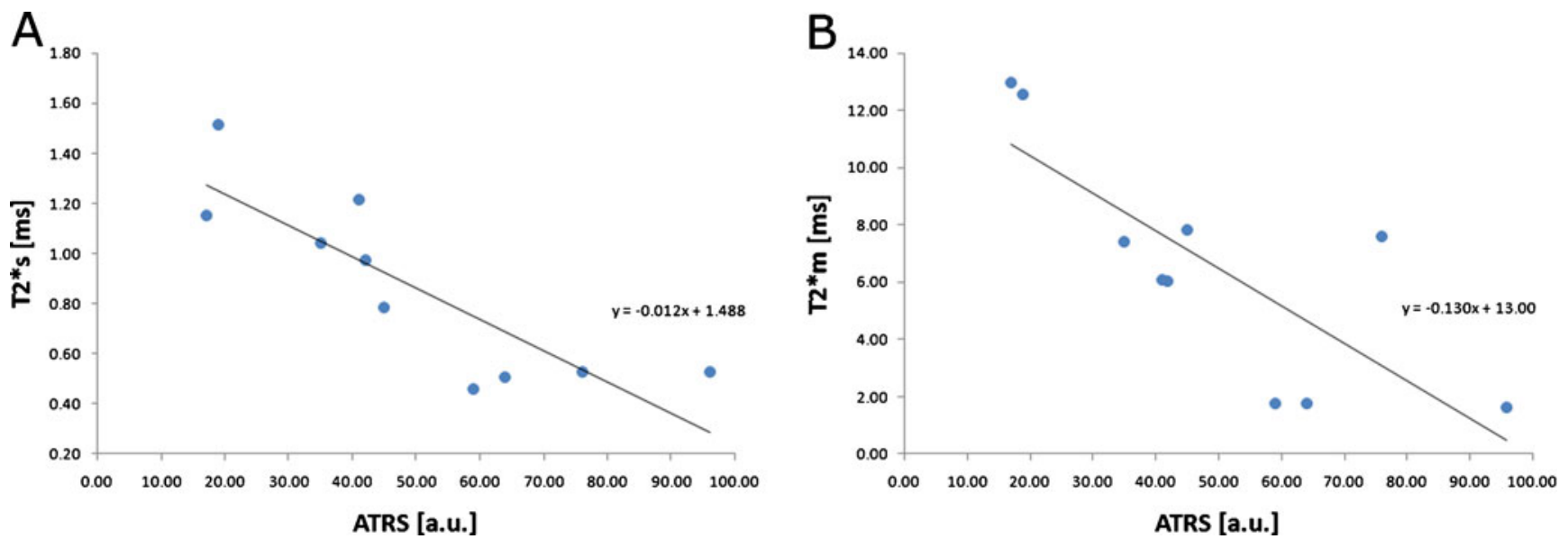

Fig. 5 a A scatter plot of the $\mathrm{T}_{2}{ }_{\mathrm{s}}$ and ATRS of ten patients. The Pearson correlation coefficient was $r=-0.846(P=0.002)$. b A scatter plot of the $\mathrm{T}_{2}{ }^{*} \mathrm{~m}$ and ATRS of ten patients. The Pearson correlation coefficient was $r=-0.786(P=0.007)$ 
fitting at the noise level measured in this study; however, no detailed simulations were performed to validate this.

The magic angle effect may bring unwanted error to $\mathrm{T}_{2} / \mathrm{T}_{2} *$ calculations. It has been shown that $\mathrm{T}_{2} *$ is approximately five times higher at $55^{\circ}$ than at $0^{\circ}$ for tendons and two times higher for entheses $[22,27]$. To minimise this problem, we positioned our patients for MR imaging such that the Achilles tendon was in a direction parallel to $\mathrm{B}_{0}$, and we tried to maintain the flexion of the ankle joint at $90^{\circ}$.

As the data in this study are presented as preliminary, further investigation with a larger patient cohort is necessary to validate the results.

The echo time range can be considered another limitation. The short $\mathrm{T}_{2}{ }^{*}$ component was sampled with the first two or three echo times, which may have introduced a greater uncertainty for the short component calculation, compared with the long component. However, the substantially higher precision (higher $\mathrm{R}^{2}$ ) of the fitting curves suggests that bi-component analysis is valid. The robustness of the method may be further improved by increasing SNR via transferring to an ultra-highfield MR device.

This new method allows better definition of the different components of the collagen fibre network within the Achilles tendon, mainly the differentiation between the slow and fast component of the $\mathrm{T}_{2}{ }^{*}$ decay, which corresponds to the free and bound proton pool within this tissue. Thus, the detection of subtle abnormalities within the Achilles tendon seems to be feasible, and the high correlation with the clinical Achilles tendon score underlines its potential as a marker for different stages of Achilles tendon disease. The ability to obtain a higher intrastructural tendon analysis may also help to define Achilles tendinopathy that is at risk for a partial tear. Finally, this technique may help in the monitoring of Achilles tendon healing after full tear surgical treatment, as it provides the opportunity to detect earlier changes as a result of abnormal healing with the risk of a re-tear of the Achilles tendon

In conclusion, the vTE is a suitable sequence for $\mathrm{T}_{2}{ }^{*}$ mapping in the human Achilles tendon in vivo, and the echo range provided by this sequence (minimum echo time, $0.8 \mathrm{~ms}$ ) is capable of two-component $\mathrm{T}_{2}{ }^{*}$ analysis. This sequence can be successfully used as an alternative to $2 \mathrm{D}$ and $3 \mathrm{D}$ UTE sequences with several benefits, such as a short imaging time, along with relatively high resolution and minimised blurring artefacts. The short component of $\mathrm{T}_{2}{ }^{*}$ provides a better marker of Achilles tendinopathy, suggesting that it reflects the changes in water content and collagen orientation more accurately than a simple mono-exponential fit.

Acknowledgement Funding support provided by the Austrian Science Fund (FWF) P 25246 B24, the Vienna Advanced Imaging Center (VIACLIC), and the Slovak Scientific Grant Agency VEGA, grant no. 2/ 0090/11.
Open Access This article is distributed under the terms of the Creative Commons Attribution Noncommercial License which permits any noncommercial use, distribution, and reproduction in any medium, provided the original author(s) and the source are credited.

\section{References}

1. Nielles-Vallespin S, Weber MA, Bock M et al (2007) 3D radial projection technique with ultrashort echo times for sodium MRI: Clinical applications in human brain and skeletal muscle. Magn Reson Med 57:74-81

2. Robson MD, Gatehouse PD, Bydder M, Bydder GM (2003) Magnetic resonance: An introduction to ultrashort TE (UTE) imaging. J Comput Assist Tomogr 27:825-846

3. Qian YX, Boada FE (2008) Acquisition-weighted stack of spirals for fast high-resolution three-dimensional ultra-short echo time MR imaging. Magn Reson Med 60:135-145

4. Song HK, Wehrli FW (1998) Variable TE gradient and spin echo sequences for in vivo MR microscopy of short T-2 species. Magn Reson Med 39:251-258

5. Ying K, Schmalbrock P, Clymer B (1995) Echo-time reduction for submillimeter resolution imaging with a $3 \mathrm{D}$ phase encode time reduced acquisition method. Magn Reson Med 33:82-87

6. Henkelman RM, Stanisz GJ, Kim JK, Bronskill MJ (1994) Anisotropy of NMR properties of tissues. Magn Reson Med 32:592-601

7. Zheng S, Xia Y (2009) Multi-components of T2 relaxation in ex vivo cartilage and tendon. J Magn Reson 198:188-196

8. Williams A, Qian Y, Golla S, Chu CR (2012) UTE-T2 * mapping detects sub-clinical meniscus injury after anterior cruciate ligament tear. Osteoarthr Cartil/OARS Osteoarthritis Res Soc 20:486-494

9. Taylor C, Carballido-Gamio J, Majumdar S, Li X (2009) Comparison of quantitative imaging of cartilage for osteoarthritis: T2, T1rho, dGEMRIC and contrast-enhanced computed tomography. Magn Reson Imaging 27(6):779-784

10. Welsch GH, Mamisch TC, Hughes T et al (2008) In vivo biochemical 7.0 Tesla magnetic resonance: preliminary results of dGEMRIC, zonal T2, and T2* mapping of articular cartilage. Investig Radiol 43:619-626

11. Mamisch TC, Hughes T, Mosher TJ et al (2012) T2 star relaxation times for assessment of articular cartilage at $3 \mathrm{~T}$ : a feasibility study. Skeletal Radiol 41:287-292

12. Juras V, Zbyn S, Pressl C et al (2012) Regional variations of T-2* in healthy and pathologic Achilles tendon in vivo at 7 Tesla: Preliminary results. Magn Reson Med 68:1607-1613

13. Robson MD, Benjamin M, Gishen P, Bydder GM (2004) Magnetic resonance imaging of the Achilles tendon using ultrashort TE (UTE) pulse sequences. Clin Radiol 59:727-735

14. Deligianni X, Bär P, Scheffler K, Trattnig S, Bieri O (2012) Highresolution Fourier-encoded sub-millisecond echo time musculoskeletal imaging at 3 Tesla and 7 Tesla. Magn Reson Med. doi:10.1002/mrm.24578 [Epub ahead of print]

15. Nilsson-Helander K, Thomee R, Gravare-Silbernagel K et al (2007) The Achilles Tendon Total Rupture Score (ATRS)-Development and validation. Am J Sports Med 35:421-426

16. Juras V, Zbyn S, Szomolanyi P, Trattnig S (2011) Regression error estimation significantly improves the region-of-interest statistics of noisy MR images. Med Phys 37:2813-2821

17. Du J, Diaz E, Carl M, Bae W, Chung CB, Bydder GM (2012) Ultrashort echo time imaging with bicomponent analysis. Magn Reson Med 67:645-649

18. Cameron IL, Short NJ, Fullerton GD (2007) Verification of simple hydration/dehydration methods to characterize multiple 
water compartments on tendon type 1 collagen. Cell Biol Int 31:531-539

19. Peto S, Gillis P, Henri VP (1990) Structure and dynamics of water in tendon from NMR relaxation measurements. Biophys J $57: 71-84$

20. Gold G, Wren T, Nayak K, Nishimura D, Beaupre G (2001) In vivo short echo time imaging of Achilles tendon. Proc Intl Soc Mag Reson Med 9:244

21. Filho GH, Du J, Pak BC et al (2009) Quantitative characterization of the Achilles tendon in cadaveric specimens: $\mathrm{T} 1$ and $\mathrm{T} 2 *$ measurements using ultrashort-TE MRI at 3 T. Am J Roentgenol 192: W117-W124

22. Du J, Chiang AJT, Chung CB et al (2010) Orientational analysis of the Achilles tendon and enthesis using an ultrashort echo time spectroscopic imaging sequence. Magn Reson Imaging 28:178-184
23. Diaz E, Chung CB, Bae WC et al (2012) Ultrashort echo time spectroscopic imaging (UTESI): an efficient method for quantifying bound and free water. NMR Biomed 25:161-168

24. Lapidus LJ, Ray BA, Hamberg P (2012) Medial Achilles tendon island flap-a novel technique to treat reruptures and neglected ruptures of the Achilles tendon. Int Orthop 36:1629-1634

25. Kearney RS, Achten J, Lamb SE, Plant C, Costa ML (2012) A systematic review of patient-reported outcome measures used to assess Achilles tendon rupture management: What's being used and should we be using it? Br J Sports Med 46:1102-1109

26. Silbernagel KG, Steele R, Manal K (2012) Deficits in heel-rise height and Achilles tendon elongation occur in patients recovering from an Achilles tendon rupture. Am J Sports Med 40:1564-1571

27. Du J, Pak BC, Znamirowski R et al (2009) Magic angle effect in magnetic resonance imaging of the Achilles tendon and enthesis. Magn Reson Imaging 27:557-564 